Series A

\author{
I. MATHEMATICA
}

380

\title{
ÜBER DIE LÖSUNG VON PARTIELLEN DIFFERENTIALGLEICHUNGEN ERSTER ORDNUNG NACH DER METHODE DER SUKZESSIVEN APPROXIMATIONEN
}

VON

MARTTI TIENARI

H E L S I N K I 1965

S U OM A L A I NEN TIEDEAKA TEM I A 
Am 10. September 1965 vorgelegt von R. Nevandinna und Oldi Lehto 


\section{Einleitung}

1.1. In dieser Arbeit wird das Problem der Lösung von der nichtlinearen vektoriellen Differentialgleichung

$$
d y=f(x, y) d x
$$

betrachtet. In (1) ist das Differential im Fréchet'schen Sinne zu verstehen. Die Räumen $R_{x}^{m}$ und $R_{y}^{n}$ der Variablen sind endlichdimensional und euklidisch.

Es handelt sich um einen neuen Beweis für den allgemeinen Existenzsatz der Lösung von Gleichung (1). Dieser Beweis bringt bezüglich der Schärfe der Annahmen nichts neues (vergl. [1] - [4]). Unsere Betrachtungen sind aber begriffsgemäss einfach, und die Konvergenz bei der Methode der sukzessiven Approximationen im mehrdimensionalen Argumentenraum wird von einem neuen Standpunkt aus untersucht. Für die Einleitung in den Problemenkreis sei auf [5] hingewiesen.

1.2. Die Behandlung der mehrdimensionalen Differentialrechnung gestaltet sich einfach, wenn man koordinatenfreie sog. absolute Bezeichnungen anwendet. In dieser Arbeit folgen wir der in der Monographie [5] eingeführten Schreibweise, die viele bekante Sätze der klassischen Analysis einer reellen Variablen zu mehreren Dimensionen verallgemeinern erlaubt.

1.3. Die Idee unseres Beweises ist kurz die folgende:

Zuerst wird die Eindeutigkeit der Lösung von Gleichung (1) bewiesen. Eine notwendige Bedingung für die Integrabilität wird danach wie üblich unter Anwendung der Symmetrie des zweiten Differentials gewonnen.

Das Neue unserer Methode besteht in der Art, wie man das Hinreichen der Integrabilitätsbedingung beweist. Dies gelingt durch direkte Betrachtung der sukzessiven Annäherungen $\left\{y_{v}(x)\right\}$. Dabei wird eine Folge von Restgliedsoperatoren $\left\{R_{v}(x)\right\}$ durch die Gleichung

$$
y_{\nu}^{\prime}(x)=f\left(x, y_{v}(x)\right)+R_{v}(x), v=0,1, \ldots,
$$


definiert. Unter Anwendung der Integrabilitätsbedingung wird dann eine Abschätzung für die Norm von $R_{\nu}(x)$ hergeleitet:

$$
\left|R_{\nu}(x)\right| \leqq M \frac{\left(3 K\left|x-x_{0}\right|\right)^{v}}{v !}, v=0,1, \ldots,
$$

wo $M=\sup |f(x, y)|$ und $K=\sup \left|f_{y}(x, y)\right|$ sind. Danach wird gezeigt, dass $\bar{y}(x)=\lim y_{v}(x)$ die Gleichung (1) erfüllt.

1.4. Um eine mehrmals vorkommende Abschätzung zu vereinfachen, schicken wir einen einfachen Hilfssatz voraus.

Lemma. Es sei $\left\{\varphi_{\nu}(r)\right\}$ eine Folge von nichtnegativen Funktionen des reellen Intervalls $0 \leqq r \leqq r_{0}$, die mit Ungleichungen

$$
\varphi_{1}(r) \leqq K, \varphi_{\nu+1}(r) \leqq M \int_{0}^{r} \varphi_{\nu}(\varrho) d \varrho, v=1,2, \ldots,
$$

beschränkt sind. Dann gilt die Abschätzung

$$
\varphi_{\nu}(r) \leqq K \frac{(M r)^{\nu-1}}{(v-1) !}, v=1,2, \ldots
$$

Der Beweis wird durch Induktion geführt. Die Abschätzung (2) gilt für $v=1$. Falls (2) für $v=n$ gültig angenommen wird, erhält man

$$
\varphi_{n+1}(r) \leqq M \int_{0}^{r} \varphi_{n}(\varrho) d \varrho \leqq M \int_{0}^{r} K \frac{(M \varrho)^{n-1}}{(n-1) !} d \varrho=K \frac{(M r)^{n}}{n !} .
$$

Also besteht (2) auch für $v=n+1$.

\section{Die lineare partielle Differentialgleichung erster Ordnung}

2.1. Wir untersuchen die Gleichung (1) unter der Anfangsbədingung )3)

$$
y\left(x_{0}\right)=y_{0} .
$$

Dafür sei im Gebiet

$$
G_{x y}=G_{x} \times G_{y}=\left\{\left|x-x_{0}\right| \leqq r_{x}\right\} \times\left\{\left|y-y_{0}\right| \leqq r_{y}\right\}
$$

folgendes angenommen:

A. Der Operator $f(x, y)$ ist in $G_{x y}$ stetig.

B. Für beliebige $y_{1}, y_{2} \in G_{y}, x \in G_{x}$ gilt die Lipschitz-Bedingung: $\left|f\left(x, y_{1}\right)-f\left(x, y_{2}\right)\right| \leqq K\left|y_{1}-y_{2}\right|$.

C. Die partiellen Ableitungen $f_{x}(x, y)$ und $f_{y}(x, y)$ sind stetig in $G_{x y}$. 
Aus der Annahme $A$ folgt es, dass in $G_{x y}$ sup $|f(x, y)|=M$ endlich ist. Weiter bemerke man, dass $B$ erfüllt ist, falls die Aussage $C$ gilt; und zwar ist die Konstante $K$ gleich der oberen Grenze der Norm $\left|f_{y}(x, y)\right|$.

2.2 Wir beweisen erstens, dass die Lösung $y(x)$ der Gleichung (1) unter Bedingung (3) eindeutig ist. Es sei hierzu angenommen, dass in $G_{x y}$ eine andere Lösung $\bar{y}(x)$ existiert. Dann ist $d[y(x)-\bar{y}(x)]=[f(x, y(x))-$ $f(x, \bar{y}(x))] d x$, und also

$$
y(x)-\bar{y}(x)=\int_{x_{0}}^{x}[f(t, y(t))-f(t, \bar{y}(t))] d t,
$$

weil $y\left(x_{0}\right)-\bar{y}\left(x_{0}\right)=0$. In (4) soll das Integral längs der Strecke $x_{0} x$ genommen werden. Setzt man auf $0 \leqq r \leqq r_{0} \leqq r_{x}$

$$
\psi(r)=\sup _{x \rightarrow x_{0} \mid \leqq r}|y(x)-\bar{y}(x)|
$$

so erhält man

$$
|y(x)-\bar{y}(x)| \leqq K \int_{x_{0}}^{x}|y(t)-\bar{y}(t)| \cdot|d t| \leqq K \int_{0}^{r} \psi(\varrho) d \varrho
$$

und daraus weiter

$$
\psi(r) \leqq K \int_{0}^{r} \psi(\varrho) d \varrho
$$

Nach der Definition ist $\psi(r) \leqq 2 r_{y}$, und so erhält man nach dem Lemma die Abschätzungen:

$$
\psi(r) \leqq 2 r_{y} \frac{(K r)^{n}}{n !}, n=0,1, \ldots .
$$

Hieraus sieht man, dass $\psi(r)=0$ und $y(x)=\bar{y}(x)$ auf $\left|x-x_{0}\right| \leqq r_{0}$ ist.

2.3 Die Gleichung (1) ist nicht allgemein integrierbar, der Operator $f(x, y)$ muss hierzu eine besondere Integrabilitätsbedingung erfüllen. Eine notwendige Bedingung finden wir, wenn wir die Symmetrie des zweiten Differentials von der Lösungsfunktion

(5) $y^{\prime \prime}(x) k h=d\left(y^{\prime}(x) h\right)=d(f(x, y(x)) h)=f_{x}^{\prime} k h+f_{y}^{\prime}\left(y^{\prime} k\right) h=f_{x}^{\prime} k h+f_{y}^{\prime}(f k) h$

fordern. Die Form (5) muss symmetrisch sein, d.h. 


$$
f_{x}^{\prime} k h+f_{y}^{\prime}(f k) h=f_{x}^{\prime} h k+f_{y}^{\prime}(f h) k .
$$

Die Bedingung (6) ist aber auch hinreichend für die Integrierbarkeit, wie wir im folgenden Kapitel beweisen werden. Für einen eindimensionalen Argumentenraum $R_{x}^{1}$ ist $(6)$ immer erfüllt. Diese Bedingung hat also nur in Räumen $R_{x}^{m}$ von mehreren Dimensionen $(m>1)$ Bedeutung.

\section{Die Methode der sukzessiven Approximationen von Picard}

3.1. Wir führen den Existenzbeweis konstruktiv durch. Die Technik ist eine unmittelbare Verallgemeinerung von der Picardschen Methode der sukzessiven Approximationen.

Wir konstruiren eine Funktionenfolge $\left\{y_{v}(x)\right\}$ :

$$
\begin{aligned}
& y_{0}(x)=y_{0}, \\
& y_{\nu+1}(x)=y_{0}+\int_{0}^{1} f\left(t, y_{v}(t)\right)\left(x-x_{0}\right) d \tau, v=0,1, \ldots,
\end{aligned}
$$

$t=x_{0}+\tau\left(x-x_{0}\right)$. Diese Folge ist wenigstens auf $\left|x-x_{0}\right| \leqq r_{0}, r_{0}=\min$ $\left[r_{x}, r_{y} / M\right]$ definiert. Aus (7) sehen wir, dass die Funktionen $y_{v}(x)$ stetig differenzierbar sind. Die Stetigkeit der Ableitungsoperatoren $y_{\nu}^{\prime}(x)$ wird durch Induktion bewiesen: Dabei schreiben wir explizite

$$
y_{v+1}^{\prime}(x) k=\int_{0}^{1}\left[\tau f_{x}^{\prime} k h+\tau f_{y}^{\prime}\left(y_{v}^{\prime} k\right) h+f k\right] d \tau,
$$

worin $f=f\left(t, y_{\mathrm{v}}(t)\right), t=x_{0}+\tau\left(x-x_{0}\right), h=x-x_{0}, y=y_{v}(t)$.

3.2. Um die Konvergenz der Folge (7) zu untersuchen, definieren wir auf dem Intervall $0 \leqq r \leqq r_{0} \leqq r_{x}$. die reelle Funktion

$$
m_{v}(r)=\sup _{\left|x-x_{0}\right| \leqq r}\left|y_{r}(x)-y_{v-1}(x)\right|, v=1,2, \ldots
$$

Aus

$$
\begin{gathered}
\left|y_{v+1}(x)-y_{v}(x)\right|=\left|\int_{x_{0}}^{x}\left[f\left(t, y_{v}(t)\right)-f\left(t, y_{v-1}(t)\right)\right] d t\right| \leqq \\
\int_{x_{0}}^{x} K\left|y_{v}(t)-y_{v-1}(t)\right| \cdot|d t| \leqq K \int_{0}^{r} m_{v}(\varrho) d \varrho
\end{gathered}
$$

erhalten wir die Abschätzung 


$$
m_{v+1}(r) \leqq K \int_{0}^{r} m_{v}(\varrho) d \varrho, v=1,2, \ldots
$$

Weil weiter $m_{1}(r)=\sup \left|y_{1}(x)-y_{0}\right| \leqq r_{y}$ ist, können wir nach dem Lemma

$$
m_{v}(r) \leqq r_{y} \frac{(K r)^{\nu-1}}{(v-1) !}
$$

schreiben. Folglich konvergiert die Folge $\left\{y_{v}(x)\right\}$ gleichmässig auf dem Intervall $\left|x-x_{0}\right| \leqq r_{0}$. Die Grenzfunktion

$$
\bar{y}(x)=\lim _{\nu \rightarrow \infty} y_{v}(x)
$$

ist stetig auf $\left|x-x_{0}\right| \leqq r_{0}$.

3.3. Wir untersuchen nun die Konvergenz der Folge $\left\{y_{\nu}^{\prime}(x)\right\}$. Dafür definieren wir einen Restgliedoperator $R_{\nu}(x)$ durch die Gleichung

$$
y_{v}^{\prime}(x)=f\left(x, y_{v}(x)\right)+R_{v}(x), v=0,1,2, \ldots,
$$

und auf dem Intervall $0 \leqq r \leqq r_{0}$ die reelle Funktion

$$
l_{v}(r)=\sup _{\left|x-x_{0}\right| \leqq r}\left|R_{v}(x)\right| \text {. }
$$

Integriert man die Gleichung (9) längs der Strecke $x_{0} x$,

$$
\int_{x_{0}}^{x} y_{v}^{\prime}(t) d t=\int_{0}^{1} f\left(t, y_{v}(t)\right)\left(x-x_{0}\right) d \tau+\int_{0}^{1} R_{v}(t)\left(x-x_{0}\right) d \tau,
$$

so wird mit Rücksicht auf (7)

$$
y_{v}(x)-y_{v+1}(x)=\int_{0}^{1} R_{v}(t)\left(x-x_{0}\right) d \tau
$$

erhalten.

3.4. Um eine Abschätzung des Restgliedes $R_{v}(x)$ zu gewinnen, werden wir eine Rekursionsformel für $R_{\nu}(x)$ herleiten. Einerseits sei dafür die Gleichung (9) in der Formel (8) eingesetzt. Wir erhalten den Ausdruck

$$
y_{v+1}^{\prime}(x) k=\int_{0}^{1}\left(\tau f_{x}^{\prime} k h+\tau f_{y}^{\prime}(f k) h+f k\right) d \tau+\int_{0}^{1} \tau f_{y}^{\prime}\left(R_{v} k\right) h d \tau,
$$


mit $f=f\left(t, y_{v}(t)\right), R=R_{v}(t), h=x-x_{0}, t=x_{0}+\tau\left(x-x_{0}\right)$. Anderseits bemerkt man, dass

$$
\begin{aligned}
f\left(x, y_{v}(x)\right) k & =\int_{0}^{1} \frac{d}{d \tau}\left[\tau f\left(t, y_{v}(t)\right) k\right] d \tau \\
& =\int_{0}^{1}\left[\tau f_{x}^{\prime} h k+\tau f_{y}^{\prime}\left(y_{\nu}^{\prime} h\right) k+f k\right] d \tau .
\end{aligned}
$$

Wenn wir in diese Formel den Ausdruck (9) von $y_{v}^{\prime}=y_{v}^{\prime}(t)$ einsetzen und die Integrabilitätsbedingung (6) anwenden, ersehen wir

$$
f\left(x, y_{v}(x)\right) k=\int_{0}^{1}\left[\tau f_{x}^{\prime} k h+\tau f_{y}^{\prime}(f k) h+f k\right] d \tau+\int_{0}^{1} \tau f_{y}^{\prime}\left(R_{v} h\right) k d \tau .
$$

Endlich subtrahieren wir diese letzte Gleichung aus (12) und erhalten die erwünschte Beziehung

$$
\begin{aligned}
R_{v+1}(x) k & =f\left(x, y_{v}(x)\right) k-f\left(x, y_{v+1}(x)\right) k \\
& +\int_{0}^{1}\left[f_{y}^{\prime}\left(R_{v} k\right) h-f_{y}^{\prime}\left(R_{v} h\right) k\right] \tau d \tau,
\end{aligned}
$$

die zur Abschätzung geeignet ist.

3.5. Aus der Gleichung (13) ergibt sich die Abschätzung

$$
\left|R_{v+1}(x) k\right| \leqq K\left|y_{v}(x)-y_{v+1}(x)\right|+\int_{0}^{1} 2\left|f_{y}^{\prime} \| R_{v, i \mid} h\right| d \tau,
$$

wenn $|k|=1$ gesetzt ist. Mit Rücksicht auf die Beziehungen (10) und (11) erhalten wir ferner

$$
\left|R_{v+1}(x) k\right| \leqq K \int_{0}^{r} l_{v}(\varrho) d \varrho+\int_{0}^{r} 2 K l_{v}(\varrho) d \varrho .
$$

Hieraus ersieht man, dass

$$
l_{\nu+1}(r) \leqq 3 K \int_{0}^{r} l_{v}(\varrho) d \varrho .
$$


Weil anderesits $l_{0}(r)=\sup \left|R_{0}(x)\right|=\sup \left|-f\left(x, y_{0}\right)\right|=M$ ist, erhalten wir nach dem Lemma die Abschätzung ${ }^{1}$ )

$$
l_{v}(r) \leqq M \frac{(3 K r)^{v}}{v !} .
$$

3.6. Wir kommen zur Frage der Konvergenz der Ableitungsfolge $\left\{y_{v}^{\prime}(x)\right\}$. Wenn die Folge $\left\{y_{v}(x)\right\}$ gegen $\bar{y}(x)$ strebt, so konvergiert $\left\{y_{v}^{\prime}(x)\right\}$ gegen $f(x, \bar{y}(x))$ : Nach Abschätzung (14) konvergiert $\left\{R_{\nu}(x)\right\}$ gleichmässig auf $\left|x-x_{0}\right| \leqq r_{0}$ gegen Null, und daraus ergibt sich

$$
\begin{aligned}
\left|y_{\nu}^{\prime}(x)-f(x, \bar{y}(x))\right| & =\left|f\left(x, y_{v}(x)\right)-f(x, \bar{y}(x))+R_{\nu}(x)\right| \\
& \leqq\left|f\left(x, y_{v}(x)\right)-f(x, \bar{y}(x))\right|+\left|R_{v}(x)\right| \\
& \leqq K\left|y_{\nu}(x)-\bar{y}(x)\right|+\left|R_{\nu}(x)\right|<\varepsilon,
\end{aligned}
$$

wenn $v>v_{\varepsilon}$. Hieraus sieht man, dass $\bar{y}(x)$ differenzierbar auf $\left|x-x_{0}\right| \leqq r_{0}$ ist und dass dort

$$
\bar{y}^{\prime}(x) k=f(x, \bar{y}(x)) k
$$

gilt.

Wir haben so das folgende Ergebnis bewiesen: Unter den Annahmen $A, B, C$ ist die Integrabilitätsbedingung (6) hinreichend für die Existenz der Lösung von der Differentialgleichung $(1)$.

Finnische Kabelwerke A. G.

Helsinki, Finnland

\section{Literatur}

[1] B̈̈снLI, G.: Über die Integrierbarkeit von Systemen partiellen, nichtlinearen Differentialgleichungen erster Ordnung. - Comment. Math. Helv. 36, $1961 / 62$, S. $245-264$.

[2] Dieudonné, J.: Foundations of modern analysis. Pure Appl. Math. 10, Academic Press, New York - London, 1960.

[3] Keller, H.H.: Ueber die Differentialgleichung erster Ordnung in normierten linearen Räumen. - Rend. Circ.Mat. Palermo II 8, 1959, S. 117-144.

[4] Louhivaara, I.S.: Ueber die Differentialgleichung erster Ordnung in normierten linearen Räumen. - Rend.Circ.Mat. Palermo II 10, 1961, S. 45- 58.

[5] Nevandinna, F. und R.: Absolute Analysis. - Grundlehren Math. Wiss. 102, Springer-Verlag, 1959.

1) Im Falle des eindimensionalen Raumes $R_{x}^{1}$ fällt aus Formel (13) das Integral der rechten Seite weg. Wir können dann in Abschätzung (14) $3 K$ durch $K$ ersetzen. 